\title{
Demonstration and Analyses of Collaboration, Coexistence, and Interoperability of Cognitive Radio Platforms
}

\author{
(Invited Paper) \\ Nolan, K.E., Sutton, P.D., Doyle, L.E. \\ Centre for Telecommunications Value-Chain Research (CTVR) \\ University of Dublin, Trinity College \\ Dublin, Rep. of Ireland \\ Telephone: $(+353)$ 1-8968443 \\ Fax: (+353) 1-8968442 \\ Email: keith.nolan@ctvr.ie, suttonpd@tcd.ie, ledoyle@tcd.ie \\ Rondeau, T.W., Le, B., Bostian, C.W., \\ Centre for Wireless Telecommunications (CWT) \\ Virginia Tech \\ Blacksburg, VA, USA \\ Telephone: (+1) 540-231-5096 \\ Fax: (+1) 540-231-3004 \\ Email: trondeau@vt.edu, binle@vt.edu, bostian@vt.edu
}

\begin{abstract}
The Centre for Telecommunications Value-Chain Research (CTVR) and the Center for Wireless Telecommunications (CWT) are undertaking a joint collaborative research project investigating the potential of different software-defined radio (SDR) and cognitive radio (CR) systems that can interoperate and co-exist in common frequency bands. This paper presents some key results from this experience so far, including an over-the-air interoperability analysis of two different SDR architectures in the same frequency band. An initial analysis of actual co-existence experiences involving primary users and secondary opportunistic spectrum users in a common frequency band is also presented.
\end{abstract}

\section{INTRODUCTION}

The use of cognitive functionality in wireless telecommunication systems and networks presents new and exciting possibilities for improving the way information is conveyed between nodes. In addition, this technology also paves the way for new wireless applications that can exploit the abilities of different cognitive radio architectures to inter-operate, coexist, and work together as a team within this network. In order to demonstrate and evaluate these possibilities, the Centre for Telecommunications Value-Chain Research (CTVR) and the Center for Wireless Telecommunications (CWT) are undertaking a joint collaborative research effort investigating these possibilities [1]. This paper describes some of the initial key outcomes of this joint collaborative research project.

The first key aim of this ongoing collaboration is the demonstration and evaluation of interoperability between two independently developed cognitive radio platforms. A second main objective is to investigate the ability of these different architectures to share a common spectrum segment and coexist in an interference-free manner. The third major objective is to develop a cognitive network and explore the potential of this network in a variety of scenarios. This paper presents some initial results from this collaborative work and examines some of the fundamental challenges experienced in the development of this practical implementation.

\section{A. Cognitive Radio and Networks}

Cognitive radio can be described as a node in a network with the abilities to form an awareness of its environment and context, make decisions and inferences from this information combined with knowledge of the user's objectives, act in a manner that attempts to accomplish the user's objectives, and finally learn from these experiences for possible use in the future [2] [4]. This cognitive functionality may have an influence on all or many of the layers in a communications stack and is not just limited to the Physical Layer (PHY) only.

\section{B. Current Scenarios and Architecture Overviews}

This section outlines the two wireless communicationsbased scenarios that were explored, leading to the experiences reported in this paper. The first scenario involves two cognitive radio systems with different architecture designs and cognitive capabilities, which can successfully interoperate with each other. In the second scenario, these same two CR architectures have reconfigured to implement two different wireless communications techniques. This scenario explores the ability of these two different CR architectures, using different wireless communication schemes, to co-exist with minimal disruption caused to each other, and exploit spectrum opportunities (unused or under-utilized whitespace frequency spectrum). The term whitespace denotes spectrum that is unused at any particular point of observation.

The first architecture involved in these tests is a GNU Radiobased platform where the parameters of the communications stack (the knobs) can be adjusted using a genetic algorithmbased cognitive control mechanism using information derived from performance metrics (the meters). This CR architecture is designed and implemented by CWT, Virginia Tech, USA [3] [7]. The second architecture design is a maximallyreconfigurable software radio system is called the Plastic Project [5], designed and implemented by CTVR, University of Dublin, Trinity College, Rep. of Ireland. The parameters 
and structure of the entire communication stack can be dynamically reconfigured according to a less complex cognitive control implementation.

\section{INTEROPERABILITY AND CO-EXISTENCE}

Interoperability in the context of this paper refers to the ability of one or more systems to provide services to and accept services from one or more other systems and to use these to enable the different systems to operate effectively together. Interoperability has increasing relevance in a world where many different wireless communications-based systems and devices exist but many of these systems cannot communicate with each other. One of the main attractions of $\mathrm{CR}$ is that it significantly increases the potential of these many different wireless communications systems to automatically detect and establish a common communications pathway. This feature is important in the Public Safety space, where different emergency services must coordinate their activities for maximum effectiveness when converging on the affected area.

Coexistence, as referred to in this paper, is the ability of two or more nodes/entities to share a common frequency band. Cognitive radio coexistence is seen as a means of helping to increase spectrum usage efficiency where two or more services can share a common frequency band without causing interference to each other; interference-free coexistence is the term denoting this ability. Exclusive spectrum usage rights for some currently licensed frequency bands can support more services, if these secondary (opportunistic) users can operate on an interference-free basis with the primary/legacy user. This is another key area in cognitive radio and dynamic spectrum access research.

\section{CTVR AND CWT COGNITIVE RADIO ARCHITECTURES}

This section provides a brief overview of the key features of the two different architectures used for the experiments in this paper.

\section{CTVR Plastic Project Architecture}

The reconfigurable core being used in this joint project is based on a multi-threaded General-Purpose-Processor platform [10] [12]. This system is known as the Plastic Project and has two main objectives in the context of this project. The first main objective of the reconfigurable core is to implement any and all of the required changes in the entire communication stack from the Application to Physical Layer (PHY). The second main objective of the core is to provide awareness information to the cognitive engine [13]. This information may include both internal radio communication system awareness and external radio environment awareness. The cognitive engine may then use this information as a key element of its cognition cycle.

A communications stack is implemented in this highly reconfigurable core as a structure containing a hierarchy of individual processing modules called Components. Considered individually, each Component can implement some or all of the functionality of a signal processing stage of the transceiver signal chain in the PHY and the majority of the other layers in the communications stack. The granularity of a Component is dependent on the designer's needs. In addition to Components existing exclusively in software, the set of Components includes hardware modules with software and firmware interfaces.

Each Component may have a number of parameters associated with it. Examples include a routing layer, which may have a cache size and beacon interval, the RF front-end operating frequencies and power levels, physical layer modulation and coding schemes, and almost any other related aspect of a communications stack that affects its operation. These parameters may be exposed by Components and reconfigured dynamically in order to alter the manner in which those Components operate.

Reconfiguration Control Mechanism: The Plastic Project reconfigurable core communications stack uses a stack manager interface to handle the reconfiguration events and cross-layer dependencies. Together with parametric reconfiguration, the Plastic Project facilitates two additional levels of reconfiguration that serve to fulfill the key objective of implementing change in the communications stack. The second level of reconfiguration is referred to as structural reconfiguration and involves the removal, addition, replacement, or interchange, of individual Components within the Plastic Project structure. Application reconfiguration forms the third and highest level of reconfiguration provided by the Plastic Project and describes the ability to replace, remove, and add entire communications stacks.

In order to fulfill the second main objective of providing awareness information to the cognitive engine, the Plastic Project maintains a shared system database referred to as a blackboard. Each Component within the Plastic Project structure is provided with access to the blackboard in order to expose awareness information that may become available. Examples of such awareness information include the current bit error rate (BER) observed within a decoding Component, the power spectral density (PSD) for the radio channel obtained through the fast Fourier transform (FFT) stage of an orthogonal frequency division multiplexing (OFDM) demodulation component or simply the current modulation scheme complexity being used by the modulation Component of a PHY transmitter chain.

\section{CWT Cognitive Radio Architecture}

The cognitive engine described in this section has been developed at the Center for Wireless Telecommunications (CWT) based in Virginia Tech [4] [3]. This engine implements the awareness-processing, decision-making, and learning elements of cognitive functionality. Specifically, this engine is capable of learning the behavior of the radio in the different environments over time and intelligently changing the communications stack to new wireless communications scenarios 


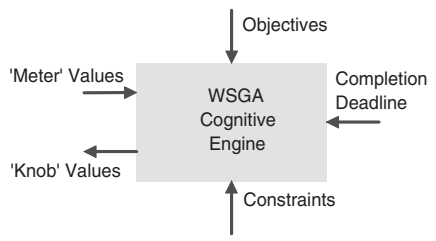

Fig. 1. Conceptual diagram of WSGA engine.

and problems efficiently based on a set of objectives and constraints. A genetic algorithm (GA) approach is used to optimize the communications stack layer parameters. Called the wireless system genetic algorithm (WSGA), this is a powerful method for exploiting the features of the highlyreconfigurable core and optimizing the operation of this core across layers in the stack. Fig. 1 is a illustrates the concept of the WSGA, where the optimisation process produces an output (the value a CR knob should adopt) that attempts to help achieve the desired objectives, based on the input parameters (meter) values and current context, according to a set of constraints (this also includes the time period in which it must be achieved by).

It is important to point out that one of the main objectives of a CR system is to devise and implement a sufficient solution within a specific time period and not an optimal solution after an implementation deadline has expired. For example, consider the example where a cognitive engine is required to deduce a suitable transmitter power level to use within a certain completion deadline ( $7 \mathrm{~ms}$ for example). The cognitive engine may decide that the optimum TX power level is $-30.003 \mathrm{dBm}$ but $10 \mathrm{~ms}$ is the time required to produce this result. A sufficient solution may be $-30 \mathrm{dBm}$ or even $-29 \mathrm{dBm}$, and to produce this result, it may only require $5 \mathrm{~ms}$. However, the value of this result is greatly increased because it is a sufficient solution achieved within the specified deadline.

In their original and most basic form, genetic algorithms (GA) were designed as single-objective search and optimization algorithms. Common to all GAs is the chromosome definition-how the data are GAs is the chromosome definition: how the data are represented; the selection mechanism for choosing the chromosomes that will survive from generation to generation; and the evaluation function used to determine the fitness of a chromosome [4]. The establishment and maintenance of effective wireless communications over a volatile communications channel requires a careful balance of the correct PHY parameters and order in which the signal chain is implemented. This balancing act can be effectively viewed as a complex multi-dimensional optimization problem, where the choice of the radio parameters on all layers affects the radio's behavior in many dimensions including (and not limited to) the bit error rate (BER), transceiver bandwidth, energy consumption, and network latency. Each of these dimensions has some relationship to the set of user and system objectives in mind. In fact, these relationships can also change in their relative importance according to the desired wireless communications application. For example, maintaining low latency in a wireless network is important for multi-player games and audio/video communications; however, for file and short message transfers, the emphasis is generally on maintaining a specified data rate and reliable transportation.

The WSGA is a multi-objective genetic algorithm (MOGA) that can influence the behavior of a reconfigurable communications stack by modeling the stack as a biological organism and optimizing its performance through genetic and evolutionary processes. In the WSGA, radio behavior is interpreted as a set of layer operation parameters defined by traits encapsulated in the genes of a chromosome. Other general radio functional parameters (including, but not limited to, payload size, power, coding techniques, encryption, equalization, number of sub-carriers, network protocol, retransmission requests, and spreading technique/code) are also identified as possible genes in the chromosome definition to cater to all of the layers in the communication stack. The WSGA analyzes the chromosome's fitness by considering a set of fitness functions defined by performance evaluations of the current communication stack. Each fitness function is weighted to represent the relative importance the user has associated with each objective. The stopping condition for deciding when an optimal or sufficient solution has been obtained is based on the user's quality of service (QoS) and application requirements. Efficiency and optimization can be subjective quantities; therefore, it is important that to know that over-maximizing is essentially a waste of radio resources such as spectrum and energy and a waste of the extra time required to complete the optimization $\operatorname{task}(\mathrm{s})$.

\section{Test Scenarios and Performance Analyses}

This section describes the interoperability and coexistence scenarios used for the initial tests involving the CWT and CTVR cognitive radio systems.

\section{A. Interoperability Experiments}

The interoperability tests involved reconfiguring the Plastic Project architecture to enable it to interpret the waveform and framing structure used by the CWT cognitive radio system. The CWT architecture was initially configured as a single carrier differential binary phase shift keying (DBPSK) based modem operating at a centre frequency of $2.41 \mathrm{GHz}$. The framing structure used is illustrated in Fig. 3. The Plastic Project platform, therefore, required a DBPSK modem and framing/de-framing Components in order to correctly interpret and produce a CWT architecture-compliant signal. The Plastic Project transceiver signal-chain was constructed using Components from an inventory of different signal-processing, modem, spectrum access, RF front-end, and sink/source reconfigurable Components, described using XML. Following this, both the CWT and CTVR's Plastic Project architecture had compatible transceiver signal chains. Fig. 2 provides an outline of the basic transceiver chain used in both instances. Although the transceiver processes used for each architecture were identical, these had non-identical implementations. 


\begin{tabular}{|c|c|c|c|c|c|}
\hline Preamble & Access Code & Header & $\begin{array}{c}\text { Packet } \\
\#\end{array}$ & Payload & CRC \\
\hline
\end{tabular}

Fig. 3. Frame structure used for the DBPSK Interoperability tests.

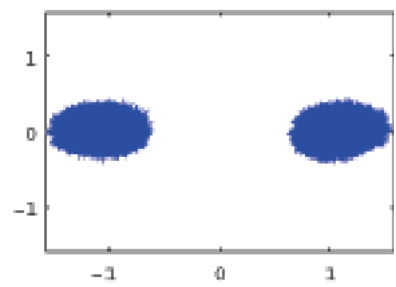

Fig. 4. Constellation diagram of received DBPSK signal.

The minimal RF front-end used for the over-the-air tests in all of the tests, and for both $\mathrm{CR}$ architectures was a $2.3 \mathrm{GHz}-$ $2.9 \mathrm{GHz}$ universal software radio peripheral (USRP) [8], which has a USB 2.0 interface and a $20 \mathrm{~mW}$ maximum TX power output. Essentially, this RF front-end performs interpolation/decimation, up/downconversion, signal amplification, and conversion to/from the analog and digital signal domains.

Fig. 4 shows the constellation diagram of received In-Phase (I) and Quadrature (Q) signal components on the $x$ and $y$ axes, respectively, following the timing recovery stage of the transceiver signal chain illustrated in Fig. 2. This indicates that the signal was received correctly, and following the demodulation and de-framing stages, the entire end to end interoperability signal-chain was completed successfully.

Fig. 5 is a constellation diagram of a sample of received quadrature phase shift keying (QPSK) signal, which could also be demodulated correctly by both CR architectures.

\section{B. Coexistence Experiments}

For the coexistence tests, the CWT cognitive radio architecture was configured to act as a narrowband DBPSK modem operating at a centre frequency of $2.41 \mathrm{GHz}$ and at a data rate of $50 \mathrm{~kb} / \mathrm{s}$. This node was designated as being a primary/legacy user. The objective here was to attempt to utilise the unused spectrum adjacent to this primary user while minimizing the possibility of interference with the primary user. Fig. 6 shows the PSD of the primary user DBPSK signal with no other users/services in the same frequency range observed in Virginia Tech, USA. The Plastic Project CR system adopted the role as a Secondary User attempting to utilize the spectrum whitespace in an opportunistic manner.

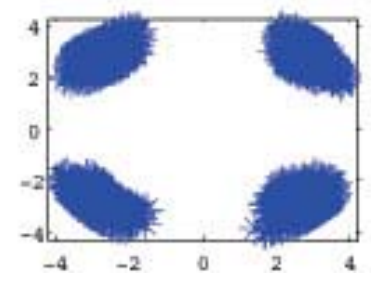

Fig. 5. Constellation diagram of received QPSK signal.

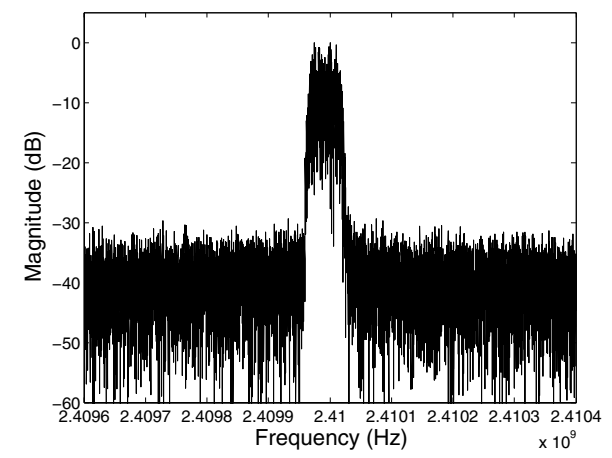

Fig. 6. Power spectral density of received primary user DBPSK signal.

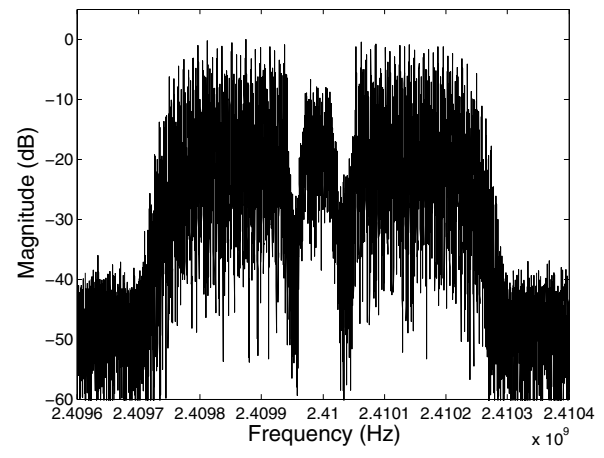

Fig. 7. Power spectral density of received primary (DBPSK) and secondary user (OFDM) signals co-existing in the same frequency band.

The Secondary User used reconfigurable orthogonal frequency division multiplexing (OFDM) [11] in order to avail of the spectrum white spaces. This required a number of tasks:

- Detection of unused frequency spectrum and avoidance of active primary user frequencies.

- Plastic Project reconfiguration to avail of the unused spectrum.

- Synchronization with other secondary user nodes.

The Plastic Project CR device reconfigured to take advantage of unused frequencies, or the spectrum whitespace, on either side of the primary user DBPSK signal. This was achieved using an energy threshold scheme based on the PSD information obtained from the OFDM FFT stage in the receiver. Fig. 7 shows the reconfigurable OFDM signal occupying unused spectrum on either side of the primary user DBPSK signal (where the original primary user signal is shown in isolation in Fig. 6). The DBPSK signal in this case was received with zero packet errors in a 2000 packet transmission test.

The ability to coexist in an interference-free manner is heavily dependent on the ability of an opportunistic user to accurately detect presence and bandwidth of an active primary/legacy user. Fig. 8 illustrates the PSD of the same DPSK primary user signal as for the first test case, but where the OFDM signal originating from the secondary user activity has incorrectly identified the region of unused spectrum and is now interfering with the primary user. In this scenario involving a 2000 packet transmission test by the primary user, 


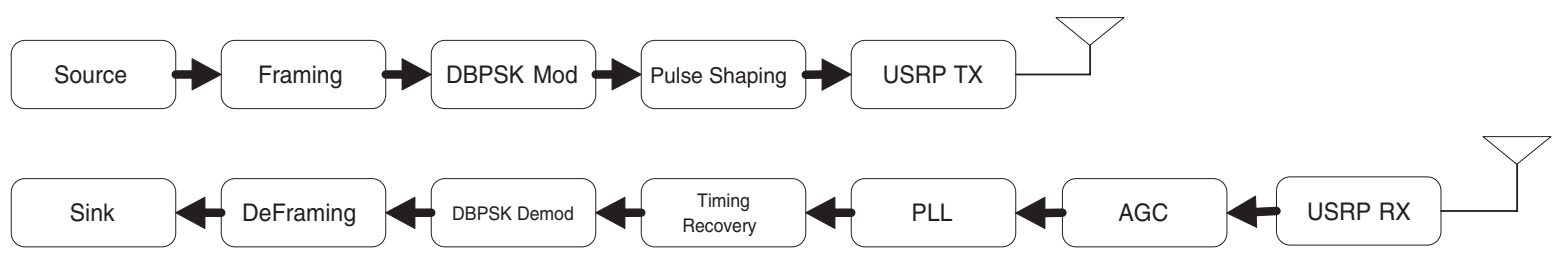

Fig. 2. Basic TXRX signal chain used for the joint CWT and CTVR Interoperability tests.

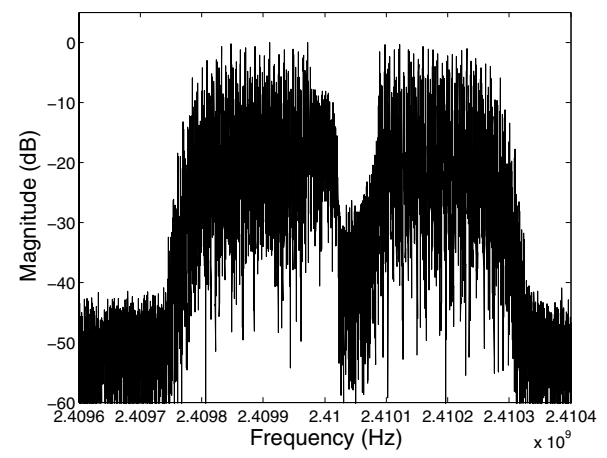

Fig. 8. Power spectral density of received primary (DBPSK) and secondary user (OFDM) signals, where the secondary user signal is interfering with the primary user.

185 packets were received incorrectly. This represents a $9.25 \%$ packet loss resulting from secondary user interference.

The main purpose of these tests was to show that the concepts of interoperability and interference-free coexistence are feasible. However, a node wishing to exploit these features should be able to accurately detect and characterize spectrum whitespace, take advantage of these opportunities, and then co-ordinate with the destination secondary user nodes. These three key challenges require robust solutions.

\section{CONCLUSIONS}

This paper has described some of the initial key results from a collaborative cognitive radio and networks project involving the Centre for Telecommunications Value-Chain Research (CTVR) in Ireland, and the Center for Wireless Telecommunications (CWT) in the U.S. These results concern the abilities of different software-defined radio (SDR) and cognitive radio (CR) systems to interoperate and co-exist in common frequency bands. The specific results presented in this paper include an analysis of successful over-the-air interoperability involving two different SDR/CR architectures in a common frequency band. Initial analysis of co-existence experiences involving primary users and secondary opportunistic spectrum users in a common frequency band have also been presented. In this case, interference-free and interfering cases were analysed.

\section{ACKNOWLEDGEMENTS}

This material is based upon work supported by Science Foundation Ireland under Grant No. 03/CE3/I405 as part of the Centre for Telecommunications Value-Chain Research
(CTVR) at Trinity College Dublin, Ireland. This work was also supported by the National Science Foundation under grants 9983463, DGE-9987586, and CNS-0519959 and by the National Institute of Justice, Office of Justice Programs, US Department of Justice under Award No. 2005-IJ-CX-K017. The opinions, findings, and conclusions or recommendations expressed are those of the author(s) and do not necessarily reflect the views of the National Science Foundation or the Department of Justice. The authors wish to thank Cara Offterdinger for her formatting and proofreading help with this paper.

\section{REFERENCES}

[1] Nolan, K. E., Rondeau T.W., Sutton, P.D., Bostian, C. W., Doyle, L.E., "A Framework For Implementing Cognitive Functionality", to appear in Proceedings of the 51st SDR Forum General Meeting and 2006 Technical Conference, Nov. 13-17, 2006, Orlando, Florida.

[2] Haykin S., "Cognitive Radio: Brain-Empowered Wireless Communications", IEEE Journal on Selected Areas in Communications, February, 2005.

[3] T. W. Rondeau, B. Le, D. Maldonado, D. Scaperoth, A. B. MacKenzie, and C. W. Bostian, "Optimization, Learning, and Decision Making in a Cognitive Engine," in Proc. SDR Forum 06 Technical Conference, Orlando, FL, 2006.

[4] Fette, B. (Editor), Cognitive Radio Technology, Newnes, Aug. 2006,ISBN: 0-7506-7952-2

[5] Doyle, L.E., Nolan, K.E., Forde T.K. et al., " A Platform for Dynamic Spectrum Experimentation ", in Proceedings of the 1st International Workshop on Technology and Policy for Accessing Spectrum (TAPAS), Boston, August 1-5 2006.

[6] T. W. Rondeau, C. J. Rieser, B. Le, and C. W. Bostian, "Cognitive Radios with Genetic Algorithms: Intelligent Control of Software Defined Radios," SDR Forum Technical Conference, Phoenix , 2004, pp. C-3 C-8.

[7] Rieser, C.J.; Rondeau, T.W.; Bostian, C.W.; Gallagher, T.M., "Cognitive radio testbed: further details and testing of a distributed genetic algorithm based cognitive engine for programmable radios," Military Communications Conference, 2004. MILCOM 2004. IEEE, vol.3, pp. 1437- 1443 Vol. 3, 31 Oct.-3 Nov. 2004.

[8] http://www.ettus.com/

[9] http://www.gnu.org/software/gnuradio/

[10] P. Mackenzie, Reconfigurable Software Radio Systems, Ph.D dissertation, University of Dublin, Trinity College, 2004.

[11] Nolan, K.E., Reconfigurable OFDM Systems, Ph.D dissertation, University of Dublin, Trinity College, 2005.

[12] Sutton, P., Doyle, L., Nolan, K.E.,"A Reconfigurable Platform for Cognitive Networks", in Proceedings of the 1st International Conference on Cognitive Radio Oriented Wireless Networks and Communications (CROWNCOM 2006), June 8-10, 2006.

[13] Nolan, K.E., Sutton, P., Doyle, L., An Encapsulation for Reasoning, Learning, Knowledge Representation, and Reconfiguration Cognitive Radio Elements, in Proceedings of the 1st International Conference on Cognitive Radio Oriented Wireless Networks and Communications (CROWNCOM 2006), Greece, June 8-10, 2006. 